\title{
Transcortical insular glioma resection: clinical outcome and predictors
}

\author{
N. U. Farrukh Hameed, MBBS, ${ }^{1}$ Tianming Qiu, MD, PhD, ${ }^{1}$ Dongxiao Zhuang, MD, PhD, ${ }^{1}$ \\ Junfeng Lu, MD, PhD, ${ }^{1}$ Zhengda Yu, MD, ${ }^{1}$ Shuai Wu, MD, ${ }^{1}$ Bin Wu, MD, ${ }^{1}$ Fengping Zhu, MD, PhD, ${ }^{1}$ \\ Yanyan Song, $\mathrm{PhD},{ }^{2}$ Hong Chen, $\mathrm{MD},{ }^{3}$ and Jinsong $\mathrm{Wu}, \mathrm{MD}, \mathrm{PhD}^{1}$
}

\begin{abstract}
${ }^{1}$ Glioma Surgery Division, Neurosurgery Department of Huashan Hospital, Fudan University; ${ }^{2}$ Department of Biostatistics, Medical School of Shanghai Jiaotong University; and 'Department of Pathology, Huashan Hospital, Fudan University, Shanghai, China
\end{abstract}

\begin{abstract}
OBJECTIVE Insular lobe gliomas continue to challenge neurosurgeons due to their complex anatomical position. Transcortical and transsylvian corridors remain the primary approaches for reaching the insula, but the adoption of one technique over the other remains controversial. The authors analyzed the transcortical approach of resecting insular gliomas in the context of patient tumor location based on the Berger-Sinai classification, achievable extents of resection (EORs), overall survival (OS), and postsurgical neurological outcome.
\end{abstract}

METHODS The authors studied 255 consecutive cases of insular gliomas that underwent transcortical tumor resection in their division. Tumor molecular pathology, location, EOR, postoperative neurological outcome for each insular zone, and the accompanying OS were incorporated into the analysis to determine the value of this surgical approach.

RESULTS Lower-grade insular gliomas (LGGs) were more prevalent (63.14\%). Regarding location, giant tumors (involving all insular zones) were most prevalent (58.82\%) followed by zone I+IV (anterior) tumors (20.39\%). In LGGs, tumor location was an independent predictor of survival $(p=0.003)$, with giant tumors demonstrating shortest patient survival $(p$ $=0.003)$. Isocitrate dehydrogenase 1 (IDH1) mutation was more likely to be associated with giant tumors $(p<0.001)$ than focal tumors located in a regional zone. EOR correlated with survival in both LGG $(p=0.001)$ and higher-grade glioma $(H G G)$ patients $(p=0.008)$. The highest EORs were achieved in anterior-zone LGGs $(p=0.024)$. In terms of developing postoperative neurological deficits, patients with giant tumors were more susceptible $(p=0.038)$. Postoperative transient neurological deficit was recorded in $12.79 \%$, and permanent deficit in $15.70 \%$ of patients. Patients who developed either transient or permanent postsurgical neurological deficits exhibited poorer survival $(p<0.001)$.

CONCLUSIONS The transcortical surgical approach can achieve maximal tumor resection in all insular zones. In addition, the incorporation of adjunct technologies such as multimodal brain imaging and mapping of cortical and subcortical eloquent brain regions into the transcortical approach favors postoperative neurological outcomes, and prolongs patient survival.

https://thejns.org/doi/abs/10.3171/2018.4.JNS18424

KEYWORDS insula; glioma; classification; surgery; prognosis; oncology

$\mathrm{T}$ HE insula is a pyramid-shaped lobe of the brain that forms part of the limbic and paralimbic systems, and brain functions ranging from cognitive to language and motor functions can be traced directly or indirectly to the insular region. ${ }^{30}$ Insular gliomas are the most common intrinsic tumors of this region, however, due to their depth and impinging neural networks, insular gliomas are dangerous to resect and have always challenged neurosurgeons. Despite this, the standard management of insular gliomas continues to remain maximum resection followed by chemoradiation therapy., ${ }^{2,10,34,37}$ The multiple risks associated with resection have forced some authors to opt for more conservative diagnostic stereotactic biopsies followed by chemoradiation therapy, ${ }^{4,29}$ while others have reported good results with microsurgical resections. ${ }^{5,17}$

The initial approach to access insular gliomas via the

ABBREVIATIONS ASPI = anterior, superior, posterior, or inferior; $\mathrm{Cl}=$ confidence interval; $\mathrm{DTI}$ = diffusion tensor imaging; $\mathrm{EOR}=$ extent of resection; $\mathrm{GTR}=$ gross-total resection; HGG = higher-grade glioma; $H R=$ hazard ratio; $I D H 1$ = isocitrate dehydrogenase 1; LGG = lower-grade glioma; $M E P=$ motor evoked potential; $O S=$ overall survival; $\mathrm{PFS}=$ progression-free survival; $\mathrm{PI}=$ proliferative index; $\mathrm{STR}=$ subtotal resection.

SUBMITTED February 12, 2018. ACCEPTED April 23, 2018.

INCLUDE WHEN CITING Published online October 19, 2018; DOI: 10.3171/2018.4.JNS18424. 
transsylvian corridor ${ }^{38}$ has improved significantly over the last two decades and continues to remain in practice. . $^{8,11}$ With advancements in awake/asleep cortical stimulation and functional brain mapping techniques, the transcortical approach to access the insula is also gaining popularity. While the choice of one technique over the other remains subjective and dependent on the surgeon's experience, there is evidence from cadaveric study ${ }^{1}$ that the transcortical corridor better exposes and is more suitable for resecting gliomas extending beyond the insula. However, there is limited conclusive evidence on the actual surgical, postsurgical, and survival implications of this approach in patients with insular glioma.

To facilitate insular tumor surgery, a number of classifications have been proposed and these have attempted to classify insular gliomas based on blood supply, ${ }^{17}$ patterns of invasiveness, ${ }^{26,37}$ white matter involvement, ${ }^{15}$ and anatomical location relative to the sylvian fissure and foramen of Monro. ${ }^{27}$ While each of these classifications has its own advantages, their value in predicting patient survival remains obscure.

In light of these complexities, we describe our transcortical surgical nuances that have been applied to a large cohort and explore how they relate to the extent of tumor resection (EOR) within each insular tumor zone based on the Berger-Sinai classification, ${ }^{27}$ overall survival (OS), postoperative neurological outcomes, and predictors.

\section{Methods \\ Patient Selection}

We analyzed 255 consecutive patients with insular glioma who underwent resection using the transcortical approach in our glioma division from March 2011 to March 2017. All surgeries were performed by the senior author (J.W.). The tumors were classified into various zones based on the Berger-Sinai classification ${ }^{27}$ using MR images. For each patient, tumor histopathology was reviewed in accordance with the 2016 WHO classification of tumors of the CNS and compared with the former 2007 WHO classification. ${ }^{12,13}$ Tumors were classified into lower-grade insular gliomas (LGGs) and higher-grade gliomas (HGGs) based on tumor grade and OS: grade II and grade III isocitrate dehydrogenase 1 (IDHI)-mutant insular tumors were denoted as LGGs, whereas grade III IDH1-wild-type and grade IV tumors were denoted as HGGs. ${ }^{13,22,23}$ Adjuvant therapy effects on patient survival were analyzed. Clinical data of patients were obtained from the Huashan Glioma Registry and onsite/telephone interviews. Informed consent was obtained from all patients preoperatively. The study was approved by the Huashan IRB.

\section{Surgical Technique}

Our transcortical approach involves awake/asleep brain mapping and multimodal brain imaging-guided resection of insular gliomas. Preoperative surgical navigation, including both structural and functional MRI, is performed. The layout of our intraoperative MRI system has been described elsewhere. ${ }^{20}$ Diffusion tensor imaging (DTI) was performed in all cases. To assess language and motor function, blood oxygen level-dependent functional MRI was also performed. Eloquent pathways and activated language and motor cortices were infused into 3D structural images using Syngo MultiModality Workplace (Siemens AG).40

The anesthetic approach was determined by presurgical evaluation of the patient's dominant hemisphere and anesthesia tolerance. Awake craniotomies (protocol described in a previous paper ${ }^{14}$ ) were preferred for most patients with dominant hemispheric tumors. Patients were placed supine with the ipsilateral shoulder elevated and placed on a rest pad, and the head tilted contralaterally to the incision site at an angle of $30^{\circ}-45^{\circ}$. A tailored frontotemporal craniotomy was performed via the pterion with the size of the bone window dependent on the extent of the tumor. Following separation of the dura, subdural strip electrodes were placed to locate the central sulcus and continuously detect the motor evoked potentials (MEPs). Language mapping was conducted for patients under awake craniotomy as described elsewhere ${ }^{14}$ (Fig. 1).

Following identification of language and motor regions, cortical incisions were made along the sylvian fissure in noneloquent regions and the opercula depending on the size, location, and opercular involvement of tumors. This was followed by subpial resection of tumors guided by structural imaging and functional navigation to minimize complications. In all cases, to preserve the pyramidal tracts and motor pathways, neuronavigation by DTI tractography was combined with continuous transcortical monitoring of MEPs and subcortical electrical stimulation mapping. Following resection, intraoperative MRI was performed to assess the EOR to guide the surgeon. In every patient, the goal of surgery was maximal tumor resection, however, tumor involvement of eloquent brain regions as well as involvement of the critical arteries limited EOR in some instances (Fig. 2). A video of the surgical technique has been presented elsewhere.?

\section{Tumor Volumetric Analysis}

EOR for each patient was measured by a neuroradiologist, and independently confirmed by a neurosurgeon (N.U.F.H., D.Z.). Tumor borders were manually delineated on MRI (FLAIR or T2-weighted sequence for LGGs as described in a previous study, ${ }^{32}$ and volume of contrastenhancing tissue on gadolinium-contrasted T1-weighted images for HGGs) in DICOM format, followed by segmentation and volumetric quantification with OsiriX software (version 5.5.2, http://www.osirix-viewer.com, Pixmeo SARL). All postoperative scans were completed within 72 hours. EOR was calculated using the following formula: [(preoperative tumor volume - postoperative tumor volume)/preoperative tumor volume] $\times 100 \%$ EOR was used to categorize tumor resections as $\geq 90 \%$ or $<$ $90 \%$ in LGGs, and $100 \%$ or $<100 \%$ in HGGs representing gross-total resection (GTR) and subtotal resection (STR), respectively.

\section{Patient Evaluation and Survival Analyses}

Patients were examined perioperatively by a senior neurosurgeon. Patients with LGG underwent postoperative follow-up at the following intervals after surgery: 3 days, 1 month, 3 months, 6 months, and every 6 months af- 

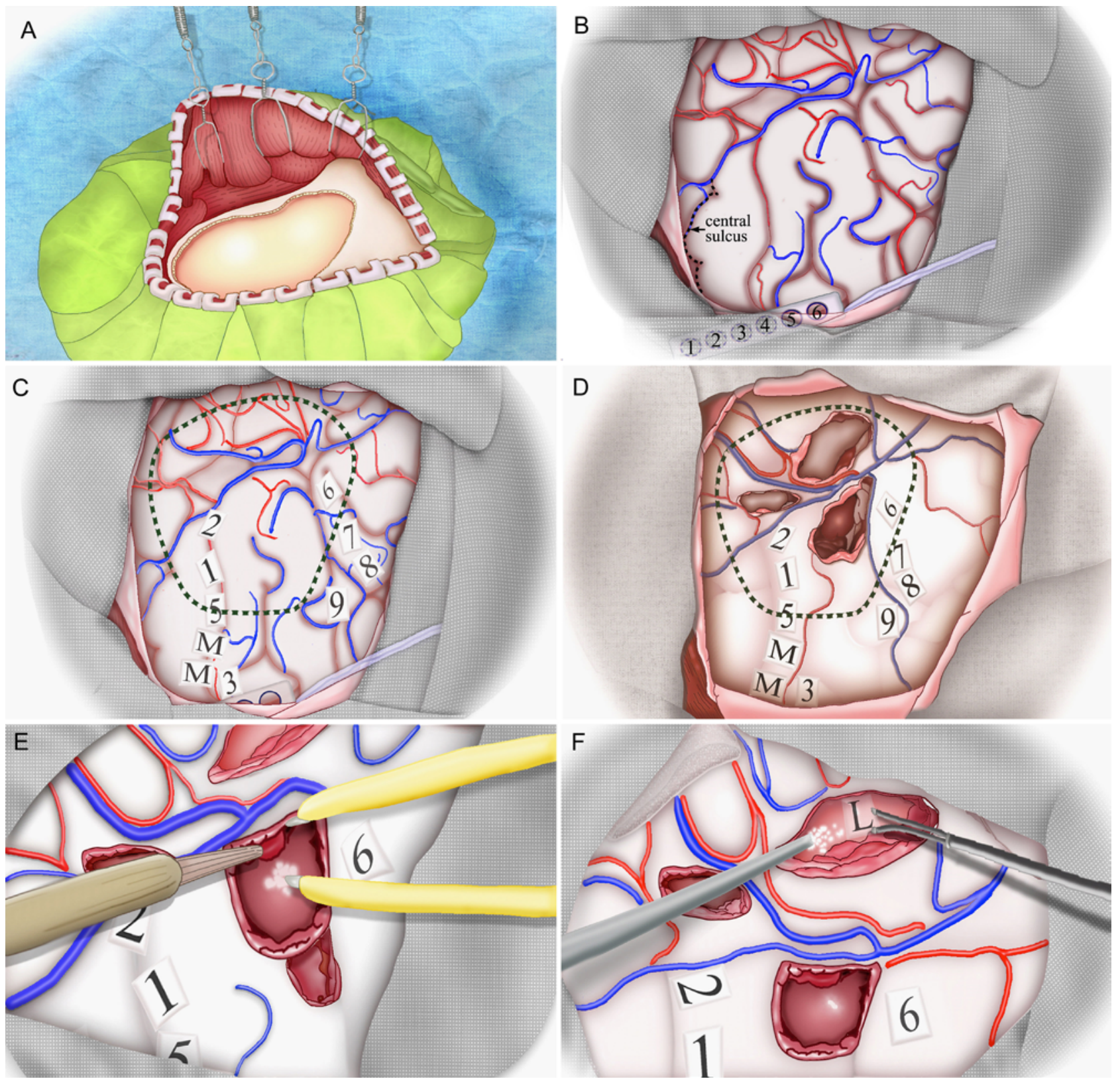

FIG. 1. A: Tailored frontotemporal craniotomy is performed via the pterion with the size of the bone window dependent on the extent of tumor. B: Following separation of the dura, subdural strip electrodes were placed to locate the central sulcus and then continuously detect the MEPs. C: Language and motor cortical mapping is performed next. Functional cortical areas are labeled with sterile tags (1, 2, 5, speech arrest; 3 , dysarthria; M, mouth motor area; $6,7,8$, 9, anomia) and covered with thin film for both visualization and protection. Under neuronavigation, the tumor border is identified and marked with 4-0 silk (dashed line). D: Cortical incisions are made, avoiding critical areas, and the incisions are made longitudinally away from the sulci to preserve the sulcal vessels. E: The tumor is gradually debunked using the cavitron ultrasonic surgical aspirator, which is set to tissue selection to sense vessels and viable tissue. F: Subcortical bipolar stimulation is conducted alongside intermittently to ensure no injury is caused to the ventral language pathway (tag "L"). Copyright Jinsong Wu. Published with permission.

terwards. Postoperative follow-up was performed 3 days, 1 month, and every 3 months afterwards for patients with HGG. Radiological assessment was performed for patients who visited the hospital for follow-up. Neurological deficits persisting at the 6-month follow-up were considered permanent, and those that resolved prior to this were considered transient. The effect of postoperative neurological deficits on survival was studied.
Based on the Berger-Sinai classification, we compared the OS between patients with giant tumors (involving all insular zones) with those with tumors involving one of these zones: zones I+IV (anterior), zones I+II (superior), zones II+III (posterior), or zones III+IV (inferior), hereafter collectively labeled as ASPI zones. These were combined because their individual sample sizes were small. Additionally, we compared the survival outcomes 


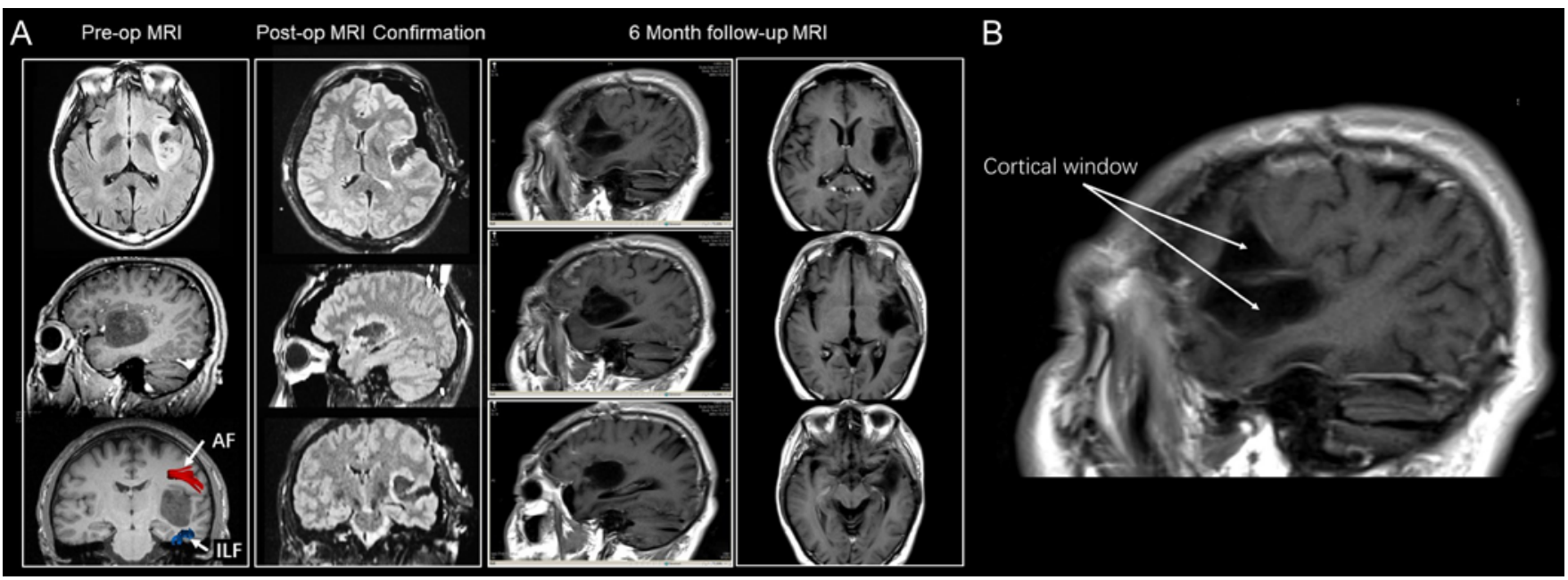

FIG. 2. A: MRI sequence showing the preoperative, postoperative, and follow-up images of a typical insular tumor. AF = arcuate fasciculus; ILF = inferior longitudinal fasciculus. B: MR image showing a cortical window created to access the insula tumor.

of patients with tumors restricted to the insular lobe only to those with insular tumors extending into neighboring lobes. For this comparison, to eliminate bias resulting from the effect of tumor location within the insula, we only included patients with giant tumors.

OS was defined as the time between initial surgery and death, whereas progression-free survival (PFS) was the time from initial surgery to demonstration of an increase in tumor volume by $25 \%$ or more on follow-up MRI, or death.

\section{Statistical Analyses}

The Kaplan-Meier method was used to estimate the OS and PFS. ANOVA was used to compare the mean EORs among various insular zones. Cox proportional hazards analysis was performed to assess the implications of various potential prognostic factors on OS. Those p values < 0.05 were considered statistically significant. IBM SPSS software (version 22, IBM Corp.) was used for data analyses.

\section{Results}

\section{Patient Demographics}

The 255 patients with insular glioma ranged in age from 20 to 78 years, and comprised 161 men and 94 women. Of these, $246(96.47 \%)$ underwent primary craniotomy and 9 (3.53\%) experienced recurrence following prior resections. One hundred and forty-five patients $(56.86 \%)$ had left-sided tumors. The most common presenting chief complaint was seizures $(41.18 \%)$. The majority of the patients $(90.80 \%)$ had a preoperative Karnofsky Performance Scale score $\geq 90$. Adjuvant therapy data were available for 247 patients, and of these patients, $76.92 \%$ received either radiotherapy (5.26\%), chemotherapy (4.21\%), or both $(90.53 \%)$. In patients with HGG, 78.72\% (74/94) were offered concurrent chemoradiation therapy in accordance with the Stupp regimen ${ }^{33}$ and clinical guidelines for diagnosis and treatment of CNS gliomas in China (2015; Table 1). ${ }^{39}$

\section{Histopathological Diagnosis}

The breakdown of tumor histological grades, based on decreasing frequency, was as follows: 120 (47.06\%) WHO grade II glioma patients, followed by 81 (31.76\%) grade IV and $54(21.18 \%)$ grade III glioma patients. We also compared the 2007 WHO classification with the 2016 WHO classification. Glioblastoma (IDH1 wild-type) was the most prevalent $(68 / 255,26.67 \%)$ followed by diffuse astrocytoma (IDH1 mutant) $(56 / 255,21.96 \%)$ and anaplastic astrocytoma (IDH1 mutant, 36/255, 14.12\%; Table 2).

IDHI wild-type status was more likely to be associated with a tumor affecting only one insular zone, whereas $\mathrm{IDH} 1$ mutation showed association with giant insular tumors (odds ratio 2.504, 95\% confidence interval [CI] $1.488-4.213, \mathrm{p}<0.001)$.

\section{Distribution of Insular Glioma Locations}

The most prevalent insular tumors were giant tumors, followed by anterior zone tumors. In the 161 patients in the LGG cohort, the majority $(\mathrm{n}=107,66.46 \%)$ had giant tumors, followed by anterior zone tumors $(\mathrm{n}=28$, $17.39 \%$ ). For the 94 patients with HGG, a similar trend was observed, with 43 patients $(45.74 \%)$ having giant and $24(25.53 \%)$ having anterior zone tumors (Table 1). The distribution of insular glioma locations was not significantly different when comparing left- and right-sided tumors. Furthermore, a similar pattern of tumor localization persisted within each histological grade.

\section{Extent of Resection}

In total, 172 patients $(67.45 \%)$ had an EOR $\geq 90 \%$. In the LGG group, 93 patients $(57.76 \%)$ had an EOR $\geq 90 \%$. In the HGG group, GTR was achieved in 40 (42.55\%) patients. In general, EOR was higher for HGGs (median EOR $98.85 \%$, interquartile range $81.51 \%-100 \%$, $95 \%$ CI $97.95 \%-100 \%, \mathrm{p}<0.001$ ) than LGGs (median EOR $95.22 \%$, interquartile range $95.25-100 \%, 95 \%$ CI $91.71 \%-$ $97.50 \%, \mathrm{p}<0.001)$. Regarding location, a significant difference was observed between the mean EORs of anterior 
TABLE 1. Patient demographics and breakdown of insular glioma mutations

\begin{tabular}{|c|c|c|c|c|c|c|c|c|}
\hline & \multirow[b]{2}{*}{ All Tumors } & \multicolumn{5}{|c|}{ Zone } & \multirow[b]{2}{*}{ LGGs } & \multirow[b]{2}{*}{ HGGs } \\
\hline & & Anterior & Superior & Posterior & Inferior & Giant Tumor & & \\
\hline No. of patients & 255 & 52 & 12 & 26 & 15 & 150 & 161 & 94 \\
\hline \multicolumn{9}{|l|}{ Age (yrs) } \\
\hline Mean & 48.2 & 49.04 & 49.25 & 50.96 & 53.1 & 46.78 & 44.48 & 54.46 \\
\hline Median & 47 & 48 & 52 & 53.5 & 51 & 46 & 45 & 56 \\
\hline Range & $20-78$ & $21-78$ & $27-69$ & $24-72$ & $34-75$ & $20-75$ & $20-74$ & $21-78$ \\
\hline \multicolumn{9}{|l|}{ Sex } \\
\hline Male & 161 & 28 & 8 & 14 & 9 & 102 & 101 & 60 \\
\hline Female & 94 & 24 & 4 & 12 & 6 & 48 & 60 & 34 \\
\hline \multicolumn{9}{|l|}{ Tumor grade } \\
\hline LGGs & 161 & 28 & 8 & 13 & 5 & 107 & - & - \\
\hline HGGs & 94 & 24 & 4 & 13 & 10 & 43 & - & - \\
\hline \multicolumn{9}{|l|}{ Adjuvant therapy } \\
\hline Radiotherapy & 10 & 1 & 1 & 1 & 1 & 6 & 10 & 0 \\
\hline Chemo & 8 & 1 & 0 & 1 & 0 & 6 & 8 & 0 \\
\hline Radiotherapy \& chemo & 172 & 37 & 4 & 17 & 8 & 106 & 98 & 74 \\
\hline No adjuvant therapy & 57 & 11 & 7 & 7 & 6 & 26 & 40 & 17 \\
\hline Not available & 8 & 2 & - & - & - & 6 & 5 & 3 \\
\hline IDH1 & 253 & 52 & 12 & 25 & 15 & 149 & 159 & 94 \\
\hline Mutation & 143 & 25 & 8 & 8 & 5 & 97 & 130 & 13 \\
\hline Wild-type & 105 & 26 & 4 & 17 & 10 & 48 & 25 & 80 \\
\hline Ambiguous & 5 & 1 & 0 & 0 & 0 & 4 & 4 & 1 \\
\hline TP53 & 226 & 46 & 10 & 24 & 15 & 131 & 141 & 85 \\
\hline Mutation & 123 & 30 & 4 & 8 & 8 & 73 & 78 & 45 \\
\hline Wild-type & 43 & 9 & 4 & 3 & 3 & 24 & 31 & 12 \\
\hline Ambiguous & 60 & 7 & 2 & 13 & 4 & 34 & 32 & 28 \\
\hline MGMT & 172 & 33 & 11 & 14 & 11 & 103 & 117 & 55 \\
\hline Methylation & 104 & 23 & 7 & 5 & 8 & 61 & 77 & 27 \\
\hline Unmethylated & 55 & 7 & 3 & 7 & 2 & 36 & 33 & 22 \\
\hline Ambiguous & 13 & 3 & 1 & 2 & 1 & 6 & 7 & 6 \\
\hline MIB-1/Ki-67 PI & 226 & 46 & 11 & 24 & 15 & 130 & 142 & 84 \\
\hline Mean (\%) & 8.26 & 9 & 8.09 & 10.83 & 11.26 & 7.18 & 4.31 & 14.93 \\
\hline Median (\%) & 5 & 8 & 3 & 5.5 & 8 & 4 & 3 & 10 \\
\hline Range (\%) & $0-60$ & $1-25$ & $1-30$ & $1-60$ & $3-30$ & $0-50$ & $0-25$ & $3-60$ \\
\hline TERT promoter & 103 & 25 & 5 & 7 & 9 & 57 & 68 & 35 \\
\hline Mutant & 38 & 9 & 1 & 2 & 4 & 22 & 26 & 12 \\
\hline Wild-type & 65 & 16 & 4 & 5 & 5 & 35 & 42 & 23 \\
\hline $1 p / 19 q$ & 128 & 32 & 5 & 8 & 9 & 74 & 97 & 31 \\
\hline Codeletion & 40 & 8 & 1 & 3 & 1 & 27 & 37 & 3 \\
\hline Intact & 88 & 24 & 4 & 5 & 8 & 47 & 60 & 28 \\
\hline
\end{tabular}

Chemo = chemotherapy; MGMT = methylguanine methyltransferase gene; $\mathrm{PI}=$ proliferative index; TERT = telomerase reverse transcriptase gene.

Values are presented as number of patients unless specified otherwise.

and giant zone tumors. The greatest EORs were achieved for anterior-zone tumors (median EOR 98.99\%, interquartile range $94.00 \%-100 \%, \mathrm{p}=0.024)$, with EOR the lowest for giant tumors (median EOR 93.61\%, interquartile range $83.50 \%-100 \%, p=0.024)$. Residual tumor was mostly located in the putamen region, posterior insular point, and superior periinsular regions in $57.83 \%$ (48/83) patients (Table 3).

\section{Overall Survival Analysis}

OS data were available for 247 patients. At last followup, $72(29.15 \%)$ patients had died of their condition, 50 
TABLE 2. Classification of 255 insular gliomas based on the old (2007) and new (2016) WHO classification

\begin{tabular}{lrlr}
\hline \multicolumn{1}{c}{ 2007 WHO Classification } & No. & 2016 WHO Classification & No. \\
\hline Diffuse astrocytoma & 75 & Diffuse astrocytoma, IDH1 mutant & 56 \\
\hline Oligodendroglioma & 32 & Diffuse astrocytoma, IDH1 wild-type & 22 \\
\hline Oligoastrocytoma & 13 & Diffuse astrocytoma, NOS & 5 \\
\hline Anaplastic astrocytoma & 42 & Oligodendroglioma, IDH1 mutant \& 1p/19q-codeleted & 25 \\
\hline Anaplastic oligodendroglioma & 6 & Oligodendroglioma, NOS & 7 \\
\hline Anaplastic oligoastrocytoma & 4 & Oligoastrocytoma, NOS & 5 \\
\hline Glioblastoma & 82 & Anaplastic astrocytoma, IDH1 mutant & 36 \\
\hline Glioblastoma-oligo & 1 & Anaplastic astrocytoma, IDH1 wild-type & 13 \\
\hline & & Anaplastic oligodendroglioma, IDH1 mutant \& 1p/19q-codeleted & 5 \\
\hline & & Glioblastoma, IDH1 mutant & 12 \\
\hline & Glioblastoma, IDH1 wild-type & 68 \\
\hline & Glioblastoma, NOS & 1 \\
\hline
\end{tabular}

NOS $=$ not otherwise specified.

(69.44\%) of whom were patients with HGG. Patients with LGG had significantly longer survival (median not reached, mean [at last follow-up] 62.68 months, 95\% CI 58.14-67.19 months, $\mathrm{p}<0.001$ ) than patients with HGG (median 17.00 months, 95\% CI 13.50-20.50 months, $\mathrm{p}<0.001$ ).

EOR was found to significantly correlate with OS in both LGGs and HGGs. In LGGs, a significantly longer mean OS was observed in patients with EOR $\geq 90 \%$ (median not reached, mean 68.51 months, 95\% CI 64.00-72.91, $\mathrm{p}=0.001$ ) than in patients with EOR $<90 \%$ (median 49.80 months, 95\% CI 48.00-51.60, $\mathrm{p}=0.001$ ). Similarly, patients with HGG and GTR had longer median survival (22.00 months, 95\% CI 16.17-27.83, $\mathrm{p}=0.008)$ than patients with STR (11.30 months, 95\% CI 8.15-14.45, $\mathrm{p}=0.008$ ).

Regarding location, for LGGs, a significantly longer OS was observed for ASPI zone tumors (median not reached, mean 73.27 months, 95\% CI 69.75-76.78 months, $\mathrm{p}=0.003$ ) than giant tumors (median 60.00 months, $95 \%$ CI 54.85-66.41 months, $\mathrm{p}=0.003)$. A similar result was noted for PFS with these patients demonstrating a longer PFS (median not reached, mean 57.55 months, 95\% CI 51.28-63.81 months, $\mathrm{p}<0.001)$ than giant tumor patients (median 29.00 months, 95\% CI 22.02-36.00 months, $\mathrm{p}<0.001$; Fig. 3). On univariate analysis, insular tumor zones continued to remain a significant predictor of OS (hazard ratio [HR] 11.105, $\mathrm{p}=0.003$ ) in LGGs. However, for HGGs, no significance was appreciated between zone involvement and OS. Additionally, HGG patients who received chemotherapy and radiotherapy had longer median OS (17.80 months, 95\% CI 14.18-21.42 months, $\mathrm{p}<0.001$ ) than those who did not (6.02 months, 95\% CI 4.00-8.40 months, $\mathrm{p}<0.001)$.

IDH1 mutation was a significant predictor of OS in LGG giant tumors, with IDH1-mutant patients demonstrating a longer survival (median not reached, mean 58.79 months, 95\% CI 52.96-64.61 months, $\mathrm{p}=0.0060$ ) than IDH1-wild-type patients (median 31.50 months, 95\% CI 8.05-55.20 months, $\mathrm{p}=0.006)$. However, in ASPI tumors, due to a smaller sample size and good overall survival (only 1 recorded death), conclusions could not be derived on the effects of IDHI mutation. For MIB-1/Ki-67, the patients were divided into 2 groups based on the MIB-1/ Ki-67 proliferative indices (PIs): $\mathrm{PI}>5 \%$ and $\mathrm{PI} \leq 5 \%$. MIB-1/Ki-67 was a significant predictor of OS (HR 0.314, $\mathrm{p}=0.013)$ in LGGs.

Tumor zone (HR 11.975, p = 0.030), EOR (HR 0.229, $\mathrm{p}=0.009)$ and IDH1-mutation (HR 4.891, $\mathrm{p}=0.008)$ remained significant predictors of OS in LGGs after controlling for multiple factors (Table 4). Putamen involvement was not a significant predictor of OS in LGGs and HGGs

TABLE 3. EOR analysis based on insular tumor locations

\begin{tabular}{lcccccccc}
\hline \multirow{2}{*}{ EOR Values } & All Tumors & Anterior & Superior & Posterior & Inferior & Giant Tumor & LGGs & HGGs \\
\cline { 3 - 7 } Median glioma vol $\left(\mathrm{cm}^{3}\right)$ & 70.45 & 56.57 & 47.22 & 64.37 & 52.92 & 80.39 & 70.47 & 67.33 \\
\hline$\geq 90 \%$ (no.) & 172 & 43 & 9 & 17 & 13 & 90 & 93 & 79 \\
\hline$<90 \%$ (no.) & 83 & 9 & 3 & 9 & 2 & 60 & 68 & 15 \\
\hline Mean (\%) & 91.60 & 95.26 & 93.69 & 91.67 & 95.19 & 89.67 & 89.05 & 95.12 \\
\hline Median (\%) & 97.95 & 98.99 & 97.49 & 98.25 & 98.89 & 93.61 & 95.22 & 98.85 \\
\hline Range (\%) & $52.61-100$ & $68.46-100$ & $71.40-100$ & $62.00-100$ & $65.81-100$ & $52.61-100$ & $55.37-100$ & $52.61-100$ \\
\hline
\end{tabular}

Residual tumor location ( $n=83$ ) was as follows: superior periinsular sulcus, $n=14(16.87 \%)$; posterior insular point, $n=16(19.28 \%)$; putamen region, $n=18(21.69 \%)$; and others, $n=35(42.17 \%)$. The "others" category includes neighboring lobes outside the insula. 

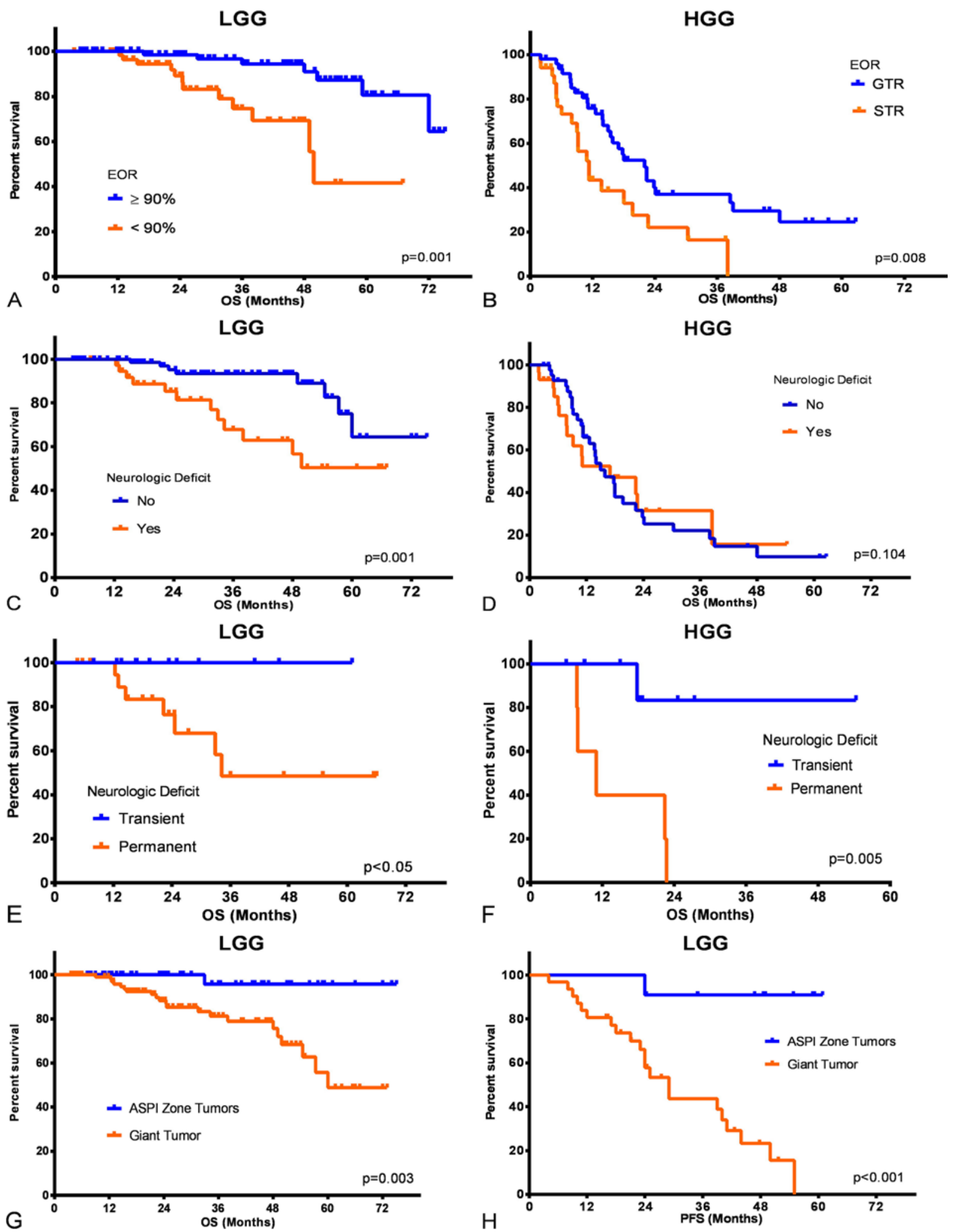

FIG. 3. A: EOR is an independent predictor of OS in both LGG and HGG patients. Patients with $E O R \geq 90 \%$ had longer survival among LGGs. B: Patients with GTR had better prognosis than those with STR among HGG. C: LGG patients who experienced no postoperative neurological deficit had better survival than those who did experience postoperative neurological deficits. D: In HGG, development of neurological deficit did not affect survival outcomes in our cohort. E and F: Patients who experienced transient neurological deficits had better OS than those who experienced permanent deficits in LGG (E) and HGG (F) patients. G and $\mathrm{H}$ : Insular tumor location was an independent predictor of OS. Longer OS $(G)$ and PFS $(H)$ were observed in the ASPI zone tumors (I+IV [anterior], I+II [superior], II+III [posterior], or III+IV [inferior]) as compared with the giant tumors (involving all insular zones). 
TABLE 4. Univariate and multivariate analyses of survival outcome in LGG patients

\begin{tabular}{|c|c|c|c|c|}
\hline & \multirow{2}{*}{$\begin{array}{c}p \\
\text { Value }\end{array}$} & \multirow[b]{2}{*}{$\mathrm{HR}$} & \multicolumn{2}{|c|}{$95 \% \mathrm{Cl}$} \\
\hline & & & Lower & Upper \\
\hline \multicolumn{5}{|l|}{ Univariate analysis } \\
\hline Sex (female) & 0.060 & 0.353 & 0.120 & 1.044 \\
\hline Age (>45 yrs) & 0.201 & 0.576 & 0.245 & 1.355 \\
\hline Zone (giant tumor) & 0.003 & 11.105 & 1.491 & 82.699 \\
\hline EOR ( $\geq 90 \%)$ & 0.001 & 0.195 & 0.072 & 0.533 \\
\hline Hemisphere (lt) & 0.879 & 1.073 & 0.432 & 2.666 \\
\hline Putamen (non-involved) & 0.515 & 0.755 & 0.324 & 1.758 \\
\hline Preop seizures (yes) & 0.984 & 1.01 & 0.378 & 2.701 \\
\hline Adjuvant therapy (given) & 0.624 & 0.738 & 0.218 & 2.499 \\
\hline IDH1 (wild-type) & 0.342 & 1.640 & 0.591 & 4.550 \\
\hline MGMT promoter (unmethylated) & 0.082 & 2.184 & 0.885 & 5.388 \\
\hline TERT promoter (wild-type) & 0.175 & 2.443 & 0.644 & 9.264 \\
\hline $1 p / 19 q$ (intact) & 0.381 & 1.707 & 0.509 & 5.726 \\
\hline TP53 (wild-type) & 0.049 & 0.314 & 0.119 & 0.827 \\
\hline MIB-1 PI ( $\leq 5 \%)$ & 0.013 & 0.314 & 0.119 & 0.827 \\
\hline \multicolumn{5}{|l|}{ Multivariate analysis } \\
\hline Sex (male) & 0.487 & 1.593 & 0.429 & 5.197 \\
\hline Age (>45 yrs) & 0.134 & 0.468 & 0.174 & 1.262 \\
\hline Zone (giant tumor) & 0.030 & 11.975 & 1.266 & 113.288 \\
\hline EOR ( $\geq 90 \%)$ & 0.009 & 0.229 & 0.076 & 0.665 \\
\hline Hemisphere (rt) & 0.300 & 0.561 & 0.188 & 1.675 \\
\hline IDH1 (wild-type) & 0.008 & 4.891 & 1.503 & 15.911 \\
\hline
\end{tabular}

Boldface type indicates statistical significance.

on both univariate and multivariate analysis. In HGGs, only EOR and adjuvant therapy were significant in predicting OS.

\section{Insular Tumors Involving Neighboring Lobes}

The tumors were not all restricted to the insular lobe only, and unsurprisingly, the majority $(203 / 255,79.61 \%)$ of them extended into one or more adjacent lobes. These lobes included the frontal $(83 / 255,32.55 \%)$, temporal $(95 / 255$, $37.25 \%$ ), and frontotemporal lobes $(25 / 255,9.80 \%)$. Six patients had tumors extending into the parietal lobe, in addition to the frontal and temporal lobes, and they were included in the frontotemporal group for statistical analysis. A similar pattern of lobar involvement was observed in both LGG and HGG groups.

Although a trend did emerge suggesting better survival in patients with tumors involving the insular lobe only, comparison between the two groups did not yield a statistically significant difference in survival.

\section{Postsurgical Neurological Follow-Up}

Complete long-term (6-month postsurgery) follow-up data were available for $172(67.45 \%)$ patients. Following surgery, $11.05 \%(19 / 172)$ patients developed language impairment, while $13.37 \%$ (23/172) developed motor impairment, of whom $68.42 \%(13 / 19)$ and $34.78 \%$ (8/23) patients, respectively, experienced complete recovery on long-term
TABLE 5. Distribution of patients who developed transient and permanent neurological deficits among 172 patients

\begin{tabular}{|c|c|c|c|}
\hline Impairment & $\begin{array}{c}\text { Transient } \\
\text { Deficit (\%) }\end{array}$ & $\begin{array}{l}\text { Permanent } \\
\text { Deficit (\%) }\end{array}$ & $\begin{array}{l}\text { Permanent Deficit } \\
\text { Specifics }\end{array}$ \\
\hline $\begin{array}{l}\text { Language } \\
\text { Giant } \\
\text { Zone I+II } \\
\text { Zone II+III } \\
\text { Zone I+IV }\end{array}$ & $\begin{array}{c}13(7.56) \\
9(5.23) \\
1(0.58) \\
3(1.74) \\
-\end{array}$ & $\begin{array}{l}6(3.49) \\
3(1.74) \\
1(0.58) \\
1(0.58) \\
1(0.58)\end{array}$ & $\begin{array}{l}\text { Lenticulostriate artery } \\
\text { infarction }(n=2, \\
1.16 \%) \text {, language } \\
\text { pathway resection ( } \mathrm{n} \\
=2,1.16 \%), M_{3} \text { infarc- } \\
\text { tion }(n=2,1.16 \%)\end{array}$ \\
\hline $\begin{array}{l}\text { Motor } \\
\text { Giant } \\
\text { Zone I+IV } \\
\text { Zone I+II } \\
\text { Zone II+III }\end{array}$ & $\begin{array}{l}8(4.65) \\
3(1.74) \\
3(1.74) \\
1(0.58) \\
1(0.58)\end{array}$ & $\begin{array}{c}15(8.72) \\
11(6.40) \\
3(1.74) \\
1(0.58) \\
-\end{array}$ & $\begin{array}{l}\text { Lenticulostriate } \\
\text { artery infarction ( } \mathrm{n} \\
=8,4.65 \%) \text {, motor } \\
\text { pathway resection ( } \mathrm{n} \\
=5,2.90 \% \text { ), long } \mathrm{M}_{2} \\
\text { perforator infarction } \\
(\mathrm{n}=2,1.16 \%)\end{array}$ \\
\hline $\begin{array}{l}\text { Language \& motor } \\
\text { Giant } \\
\text { Zone I+IV } \\
\text { Zone I+II } \\
\text { Zone II+III }\end{array}$ & $\begin{array}{l}1(0.58) \\
1(0.58) \\
- \\
- \\
-\end{array}$ & $\begin{array}{l}6(3.49) \\
3(1.74) \\
1(0.58) \\
1(0.58) \\
1(0.58)\end{array}$ & $\begin{array}{l}\text { Lenticulostriate artery } \\
\text { infarction ( } n=1 \text {, } \\
0.58 \%) \text {, motor path- } \\
\text { way resection ( } n=2 \text {, } \\
1.16 \%), M_{2} \text { infarction } \\
(n=3,1.74 \%)\end{array}$ \\
\hline
\end{tabular}

follow-up. Both motor and language neurological deficits arose in $4.1 \%$ (7/172) of the patients (Table 5).

The majority of the patients $(16 / 19,84.21 \%)$ who developed postoperative language deficits either had a giant or posterior insular tumor. By comparison, most patients developing postoperative motor deficits either had a giant or anterior insular tumor $(20 / 23,86.96 \%)$. Generally, patients with giant tumors were at a higher risk of developing postoperative neurological deficits (relative risk 1.58, $\mathrm{p}=$ $0.038)$.

In addition to these deficits, over the course of longterm follow-up, $8.97 \%$ (13/145) postoperatively asymptomatic patients developed late-onset language or motor impairment. Among these, $76.92 \%(10 / 13)$ had a giant tumor. All patients who developed language impairment had dominant-sided tumors, whereas for patients with motor impairment, tumor location was evenly distributed between dominant and nondominant hemispheres. Overall, for LGG, transient deficit was recorded in $9.84 \%(12 / 122)$ and permanent deficit in $17.21 \%(21 / 122)$ patients. For HGG, transient deficit was recorded in 20\% (10/50) and permanent deficit in $12 \%$ (6/50). In patients who developed permanent deficits, the incidence of postoperative infarction was $66.67 \%$ (18/27). Lenticulostriate artery infarction was detected in $40.74 \%$ (11/27) patients (Table 5).

Patients with transient deficit showed better survival than those with permanent deficits in both LGG and HGG $(\mathrm{p}<0.05)$. LGG patients who did not develop any postsurgical neurological deficit had a better OS (median not reached, mean 66.89 months, 95\% CI 61.81-71.96 months, $\mathrm{p}<0.001)$ than those who did develop some form of postoperative neurological deficit (median 48.00 months, 95\% CI 26.80-69.20 months, $\mathrm{p}<0.001)$. Predictably, patients who developed both language and motor neurological deficits had the worst survival (median 41.50 months, 95\% 
CI 27.05-58.00 months, $\mathrm{p}=0.001)$. In HGGs, development of postoperative neurological deficits did not affect OS (Fig. 3).

\section{Discussion}

Due to the complexity of insular glioma surgeries, the selection of the most appropriate surgical technique for patients that allows maximal tumor resection while minimizing morbidity continues to remain problematic. Our large cohort study shows that the transcortical corridor, when combined with multimodal brain imaging and adequate mapping of cortical and subcortical eloquent brain regions, is an effective surgical approach for maximizing EOR in all insular zones and minimizing postoperative morbidity.

The transsylvian approach for accessing insular gliomas, first described by Yaşargil et al. ${ }^{38}$ and Hentschel and Lang, ${ }^{8}$ has developed over the years ${ }^{35}$ and continues to remain appealing to neurosurgeons due to its uniqueness. In this approach, a transsylvian corridor is created to access the insula by opening the superficial and deep sylvian cisterns. However, multimodal imaging technology and techniques for cortical and subcortical mapping of brain function have recently advanced and dramatically expanded surgical possibilities, making transcortical resection of insular tumors more popular. In this approach, opercular eloquence is mapped and opercular "windows" in functionally silent cortical surfaces are created through which tumor is resected. In this approach, dominant posterior zone tumors are challenging to resect because the cortical windows to these tumors, at the parietotemporal junction, are often eloquent. ${ }^{4,6}$ Nevertheless, they can be approached through tailored corticotomies of the postcentral and/or supramarginal gyri.

In patients with eloquent opercular cortex precluding transcortical resection, the transsylvian approach is very suitable. A notable example is the frontal operculum that harbors Broca's area (pars opercularis and triangularis) that is critical to speech. Also, for smaller tumors, this approach is useful because the insula can be reached without the need for performing cortical incisions. However, as our results demonstrate, most insular tumors $(79.61 \%)$ extend into neighboring brain regions, and to maximally resect these tumors a greater degree of surgical freedom via widened transsylvian corridors would be required. Unfortunately, the need to preserve the superficial sylvian veins bridging the sylvian fissure limits the surgical freedom of the transsylvian approach and the possible EOR. In fact, a cadaveric study ${ }^{1}$ explored the possibility of cutting these sylvian bridging veins to further widen the transsylvian surgical corridor for resecting larger tumors but found it unsafe in $30 \%$ of patients. Additionally, the narrow sylvian cistern greatly limits the resection of posterior insular tumors, and in some instances, this approach could be insufficient to even expose these tumors. ${ }^{1}$ However, the median EOR $(98.25 \%)$ achieved by our transcortical approach for posterior zone insular tumors was not limited by tumor location and was as high as that of other zones with good postoperative outcome.

Despite the advantages of resecting insular gliomas via the transsylvian corridor stemming from cortical sparing and a recent study mentioning no associated major surgical or neurological complications with it, ${ }^{25}$ some studies have documented complications in as many as 30\% of patients undergoing this approach, and most of these complications arise from opercular retraction causing edema and ischemia of $\mathbf{M}_{3}$ branches. ${ }^{8,24,38}$ This approach also involves manipulation of delicate arteries within the sylvian fissure that can risk ischemic postoperative neurological deficits. ${ }^{3,4,6}$ By comparison, the transcortical approach, when used with adjuncts as described in our surgical technique, has a significantly better morbidity profile as demonstrated by our results and others. ${ }^{4,8,27,28}$

Previously, Yaşargil et al. ${ }^{38}$ and Mandonnet et al. ${ }^{15}$ have classified insular gliomas anatomically. In this study however, we used the Berger-Sinai classification. ${ }^{27} \mathrm{Be}-$ cause most tumors involving a single zone had a majority of tumor body located outside the insula, we only included patients with more than one insular zone clearly involved, with the main tumor body in the insula. Among these zones, giant LGG tumors demonstrated worst patient survival and had the lowest EOR. The greater preoperative tumor volume and risk associated with their surgical resection may have limited their EOR, and consequently, patients' OS. By comparison, when LGGs were limited to one of the ASPI zones, EOR and OS improved significantly. Moreover, the PFS for these patients was almost twice that of patients with giant tumors. Based on these results, a more aggressive follow-up and adjuvant therapy regimen could be suggested for patients with LGG giant tumors. For HGGs, results suggest that the more aggressive tumor pathology of HGGs favoring invasiveness and penetration of anatomical barriers limits the applicability of this classification in predicting survival. Recently, Wang et al. ${ }^{36}$ proposed a putamen-based classification that could predict patient survival. However, our results do not concur with this. A possible explanation could be that Wang et al. did not clarify tumor involvement of other brain zones besides the putamen, and this could have influenced outcome.

We found EOR to vary significantly among the various insular zones. Due to their close proximity to pyramidal tracts, posterior zone tumors had lesser EORs than anterior zone tumors, while giant zone tumors had the lowest EORs because maximum effort was directed at preserving function during their resection. Resection of tumor at the posterior insular point, superior periinsular sulcus, and the putamen region (in close proximity to lenticulostriate arteries) presents difficulty; therefore, insular residual tumor is most likely to be found here. This also explains the general lower mean EOR recorded for the posterior and superior insular region tumors. In the HGG group, median EOR was higher because of relatively visible blood brain barrier disrupting tumor margins. In addition, these tumors were resected more aggressively. The EORs for various insular zones from our large cohort could serve as a reference point for surgeons adopting the transcortical approach to estimate possible tumor resections for tumors in these zones and the accompanying OS.

EOR remains the most important determinant of OS, and this point is further supported by our adjuvant therapy survival analysis. Based on the National Comprehensive Cancer Network guidelines and clinical guidelines for di- 
agnosis and treatment of central nervous system gliomas in China (2015), ${ }^{39}$ LGG patients with GTR are followed up closely, while those with STR receive adjuvant therapy. Adjuvant therapy administration improved OS in patients with STR, and this explains why a statistically significant difference was not observed between LGG patients receiving and not receiving adjuvant therapy. However, in HGG patients, those who received adjuvant therapy demonstrated a better OS. It is worth noting that the few patients who did not or opted not to receive adjuvant therapy already had poor postoperative recovery, which also explained their short survival.

A comparison between the 2007 and 2016 WHO classifications was also performed, showing that grade III tumors could now be more easily distinguished as lower or higher grade, which facilitates clinical decision-making in cases requiring more aggressive treatment. While some studies $^{18}$ have shown differences in survival between grade II and III tumors, others have not. ${ }^{19,23}$ Therefore, we classified grade III tumors into lower and higher grade based on their survivals. Analysis of mutations prevalent in our cohort revealed the IDH1-mutant form to be more prevalent among giant tumors. Moreover, IDH1-mutant giant tumor patients exhibited longer survival. By contrast, IDHI wild-type was more prevalent among ASPI tumor patients, yet their OS was longest. These data suggest that in addition to influencing patient prognosis, IDH1 mutation could also be a potential predictor of tumor growth tendencies. The improved survival in ASPI IDH1-wild-type patients could be due to smaller tumor size and improved EOR as compared to giant tumors with total insular involvement. A comparison between $I D H 1$-mutant and IDHI-wild-type statuses could not be made for the various ASPI zone tumor patients because of a smaller sample size.

The prevalence of postoperative transient deficits ranges from $14 \%$ to $59 \%$ and that of permanent deficit from $0 \%$ to $20 \% .^{4,9,31}$ In our study, postoperative transient deficit was recorded in $12.79 \%$ and permanent deficit in $15.70 \%$ of patients. Also, tumor recurrence symptoms included new late-onset deficits. Regarding location, the larger transcortical window required to resect giant tumors could explain their relatively poorer prognosis. The results indicate that tumor location can predict the likelihood of developing postoperative neurological deficit in patients with insular glioma. Insular surgeries resulting in motor morbidity have been associated with a poorer prognosis. ${ }^{16,21}$ Similarly, we have shown the morbidity associated with development of neurological deficits.

In this study, investigation of survival among the various individual insular tumor zones could not be performed due to inadequate sample size. In addition, complete clinical follow-up data were not available for a significant number of our patients. These limitations warrant further investigation in this direction.

\section{Conclusions}

The transcortical surgical approach, when combined with adjuncts, maximizes EOR and improves postoperative outcomes in patients with insular glioma. Moreover, in transcortical insular tumor resection, the Berger-Sinai classification independently predicts survival, EOR, and postoperative complications, which aids in clinical decision-making.

\section{Acknowledgments}

This project was sponsored by The National Key Technology R\&D Program of China (grant no. 2014BAI04B05) and The Foundation of National Natural Science Fund, China (grant nos. 81672476 and 81401136).

\section{References}

1. Benet A, Hervey-Jumper SL, Sánchez JJ, Lawton MT, Berger MS: Surgical assessment of the insula. Part 1: surgical anatomy and morphometric analysis of the transsylvian and transcortical approaches to the insula. J Neurosurg 124:469-481, 2016

2. Delion M, Mercier P: Microanatomical study of the insular perforating arteries. Acta Neurochir (Wien) 156:1991-1998, 2014

3. Duffau H: A new concept of diffuse (low-grade) glioma surgery. Adv Tech Stand Neurosurg 38:3-27, 2012

4. Duffau H: A personal consecutive series of surgically treated 51 cases of insular WHO Grade II glioma: advances and limitations. J Neurosurg 110:696-708, 2009

5. Duffau H, Capelle L, Lopes M, Faillot T, Sichez JP, Fohanno D: The insular lobe: physiopathological and surgical considerations. Neurosurgery 47:801-811, 2000

6. Duffau H, Moritz-Gasser S, Gatignol P: Functional outcome after language mapping for insular World Health Organization Grade II gliomas in the dominant hemisphere: experience with 24 patients. Neurosurg Focus 27(2):E7, 2009

7. Hameed NUF, Zhu Y, Qiu T, Wu J: Awake brain mapping in dominant side insular glioma surgery: 2-dimensional operative video. Oper Neurosurg (Hagerstown) [epub ahead of print], 2018

8. Hentschel SJ, Lang FF: Surgical resection of intrinsic insular tumors. Neurosurgery 57 (1 Suppl):176-183, 2005

9. Hervey-Jumper SL, Li J, Osorio JA, Lau D, Molinaro AM, Benet A, et al: Surgical assessment of the insula. Part 2: validation of the Berger-Sanai zone classification system for predicting extent of glioma resection. J Neurosurg 124:482488,2016

10. Ius T, Pauletto G, Isola M, Gregoraci G, Budai R, Lettieri $\mathrm{C}$, et al: Surgery for insular low-grade glioma: predictors of postoperative seizure outcome. J Neurosurg 120:12-23, 2014

11. Lang FF, Olansen NE, DeMonte F, Gokaslan ZL, Holland EC, Kalhorn C, et al: Surgical resection of intrinsic insular tumors: complication avoidance. J Neurosurg 95:638-650, 2001

12. Louis DN, Ohgaki H, Wiestler OD, Cavenee WK, Burger PC, Jouvet A, et al: The 2007 WHO classification of tumours of the central nervous system. Acta Neuropathol 114:97-109, 2007

13. Louis DN, Perry A, Reifenberger G, von Deimling A, Figarella-Branger D, Cavenee WK, et al: The 2016 World Health Organization Classification of Tumors of the Central Nervous System: a summary. Acta Neuropathol 131:803-820, 2016

14. Lu J, Wu J, Yao C, Zhuang D, Qiu T, Hu X, et al: Awake language mapping and 3-Tesla intraoperative MRI-guided volumetric resection for gliomas in language areas. J Clin Neurosci 20:1280-1287, 2013

15. Mandonnet E, Capelle L, Duffau H: Extension of paralimbic low grade gliomas: toward an anatomical classification based on white matter invasion patterns. J Neurooncol 78:179-185, 2006

16. McGirt MJ, Mukherjee D, Chaichana KL, Than KD, Weingart JD, Quinones-Hinojosa A: Association of surgically 
acquired motor and language deficits on overall survival after resection of glioblastoma multiforme. Neurosurgery 65:463-470, 2009

17. Moshel YA, Marcus JDS, Parker EC, Kelly PJ: Resection of insular gliomas: the importance of lenticulostriate artery position. J Neurosurg 109:825-834, 2008

18. Ohgaki H, Kleihues P: The definition of primary and secondary glioblastoma. Clin Cancer Res 19:764-772, 2013

19. Ohgaki H, Kleihues P: Population-based studies on incidence, survival rates, and genetic alterations in astrocytic and oligodendroglial gliomas. J Neuropathol Exp Neurol 64:479-489, 2005

20. Qiu TM, Yao CJ, Wu JS, Pan ZG, Zhuang DX, Xu G, et al: Clinical experience of $3 \mathrm{~T}$ intraoperative magnetic resonance imaging integrated neurosurgical suite in Shanghai Huashan Hospital. Chin Med J (Engl) 125:4328-4333, 2012

21. Rahman M, Abbatematteo J, De Leo EK, Kubilis PS, Vaziri $\mathrm{S}$, Bova F, et al: The effects of new or worsened postoperative neurological deficits on survival of patients with glioblastoma. J Neurosurg 127:123-131, 2017

22. Reuss DE, Kratz A, Sahm F, Capper D, Schrimpf D, Koelsche C, et al: Adult IDH wild type astrocytomas biologically and clinically resolve into other tumor entities. Acta Neuropathol 130:407-417, 2015

23. Reuss DE, Mamatjan Y, Schrimpf D, Capper D, Hovestadt V, Kratz A, et al: IDH mutant diffuse and anaplastic astrocytomas have similar age at presentation and little difference in survival: a grading problem for WHO. Acta Neuropathol 129:867-873, 2015

24. Rey-Dios R, Cohen-Gadol AA: Technical nuances for surgery of insular gliomas: lessons learned. Neurosurg Focus 34(2):E6, 2013

25. Safaee MM, Englot DJ, Han SJ, Lawton MT, Berger MS: The transsylvian approach for resection of insular gliomas: technical nuances of splitting the Sylvian fissure. J Neurooncol 130:283-287, 2016

26. Saito R, Kumabe T, Kanamori M, Sonoda Y, Tominaga T: Insulo-opercular gliomas: four different natural progression patterns and implications for surgical indications. Neurol Med Chir (Tokyo) 50:286-290, 2010

27. Sanai N, Polley MY, Berger MS: Insular glioma resection: assessment of patient morbidity, survival, and tumor progression. J Neurosurg 112:1-9, 2010

28. Schramm J, Aliashkevich AF: Surgery for temporal mediobasal tumors: experience based on a series of 235 patients. Neurosurgery 60:285-295, 2007

29. Shankar A, Rajshekhar V: Radiological and clinical outcome following stereotactic biopsy and radiotherapy for low-grade insular astrocytomas. Neurol India 51:503-506, 2003

30. Shelley BP, Trimble MR: The insular lobe of Reil-its anatamico-functional, behavioural and neuropsychiatric attributes in humans - a review. World J Biol Psychiatry 5:176-200, 2004

31. Simon M, Neuloh G, von Lehe M, Meyer B, Schramm J: Insular gliomas: the case for surgical management. J Neurosurg 110:685-695, 2009
32. Smith JS, Chang EF, Lamborn KR, Chang SM, Prados MD, Cha $S$, et al: Role of extent of resection in the long-term outcome of low-grade hemispheric gliomas. J Clin Oncol 26:1338-1345, 2008

33. Stupp R, Mason WP, van den Bent MJ, Weller M, Fisher $\mathrm{B}$, Taphoorn MJ, et al: Radiotherapy plus concomitant and adjuvant temozolomide for glioblastoma. $\mathbf{N}$ Engl J Med 352:987-996, 2005

34. Tanriover N, Rhoton AL Jr, Kawashima M, Ulm AJ, Yasuda A: Microsurgical anatomy of the insula and the sylvian fissure. J Neurosurg 100:891-922, 2004

35. Wang P, Wu MC, Chen SJ, Xu XP, Yang Y, Cai J: Microsurgery resection of intrinsic insular tumors via transsylvian surgical approach in 12 cases. Cancer Biol Med 9:44-47, 2012

36. Wang Y, Wang Y, Fan X, Li S, Liu X, Wang J, et al: Putamen involvement and survival outcomes in patients with insular low-grade gliomas. J Neurosurg 126:1788-1794, 2017

37. Yaşargil GM, Krisht AF, Türe U, Al-Mefty O, Yaşargil DCH: Microsurgery of insular gliomas. Part I: surgical anatomy of the sylvian cistern. Contemp Neurosurg 24:1-8, 2002

38. Yaşargil MG, von Ammon K, Cavazos E, Doczi T, Reeves JD, Roth P: Tumours of the limbic and paralimbic systems. Acta Neurochir (Wien) 118:40-52, 1992

39. Zhou L, Mao Y, Wang R, Bian X, Chen L, Chen S, et al: [Clinical guidelines for diagnosis and treatment of central nervous system gliomas in China (2015).] Zhonghua Yi Xue Za Zhi 96:485-509, 2016 (Chinese)

40. Zhu FP, Wu JS, Song YY, Yao CJ, Zhuang DX, Xu G, et al: Clinical application of motor pathway mapping using diffusion tensor imaging tractography and intraoperative direct subcortical stimulation in cerebral glioma surgery: a prospective cohort study. Neurosurgery 71:1170-1184, 2012

\section{Disclosures}

The authors report no conflict of interest concerning the materials or methods used in this study or the findings specified in this paper.

\section{Author Contributions}

Conception and design: J Wu, Hameed, Zhuang. Acquisition of data: Hameed, Yu, S Wu, B Wu, Chen. Analysis and interpretation of data: J Wu, Hameed, Qiu, Zhuang, Chen. Drafting the article: Hameed. Critically revising the article: J Wu, Qiu, Zhuang. Reviewed submitted version of manuscript: Qiu, Lu. Approved the final version of the manuscript on behalf of all authors: J Wu. Statistical analysis: Hameed, Song. Administrative/technical/material support: Zhuang, Lu, Zhu.

\section{Correspondence}

Jinsong Wu: Huashan Hospital, Fudan University, Shanghai, China.wjsongc@126.com; wujinsong@huashan.org.cn. 